\title{
Optimal Power Allocation in Downlink Non-Orthogonal Multiple Access (NOMA)
}

\author{
Wajd Fahad Alghasmari ${ }^{1}$, Laila Nassef ${ }^{2}$ \\ Department of Computer Science \\ Faculty of Computing and Information Technology \\ King Abdulaziz University, Jeddah \\ Saudi Arabia
}

\begin{abstract}
Fifth generation of wireless cellular networks promise to enable better services anytime and anywhere. Nonorthogonal multiple access (NOMA) stands as a suitable multiple accessing scheme due to the ability to allow multiple users to share the same radio resource simultaneously via different domains (power, code, etc.). Through the introduced power domain, users multiplexed at the radio resource within different power levels. This paper studies power allocation in downlink NOMA, an optimization problem formulated that aims to maximize the system's sum rate. To solve the problem, a genetic algorithm based power allocation (GAPA) was proposed that uses genetic algorithm (GA) that employs heuristics to search for suitable solutions. The performance of the proposed power allocation algorithm compared with full search power allocation (FSPA) that gives an optimal performance. Results show that GAPA reaches a performance near to FSPA with lower complexity. In addition, GAPA simulated with various user paring algorithms. Channel state sorting based user pairing with GAPA achieves the best performance comparing to random user pairing algorithm and exhaustive user pairing.
\end{abstract}

Keywords-Non-orthogonal multiple access; power allocation; genetic algorithm; user pairing

\section{INTRODUCTION}

Conventional orthogonal multiple access (OMA) takes an incredible role starting from the first generation of wireless cellular networks $(1 \mathrm{G})$ to the fourth generation $(4 \mathrm{G})$ that uses orthogonal frequency division multiple access (OFDMA) for downlink network and single carrier (SC-OFDMA) for the uplink network [1]. Although OMA provides a various number of advantages to the network, it cannot handle massive connectivity under the diversity of quality of services (QoS) demands by users. Fairness, scarcity of the spectrum, and increasing number of connected devices add additional obstacles for OMA [2]. Non-orthogonal multiple access (NOMA) introduced for the fifth generation (5G) [3] [4]. NOMA represented as a suitable multiple accessing scheme due to the ability to handle an increased number of users and boosting the performance of the system such as spectrum efficiency [5]. Various schemes of non-orthogonal multiple access proposed such as Multiple User Shared Multiple Access (MUSA), Interleaver Division Multiple Access (IDMA), Spare
Code Multiple Access (SCMA), and Pattern Division Multiple Access (PDMA). These schemes are divided into several categorize based on their properties [6], where MUSA and Resource Spread Multiple Access (RSMA) use spreading sequences. Otherwise, a structured coding matrix is used in SCMA and PDMA where IDMA based on interleaver and NOMA schemes is build based on the power domain.

In power domain NOMA, multiple users assigned to one resource block (RB) through different power levels utilizing superposition coding (SC) and successive interference cancelation (SIC) [7]. Channel condition plays a critical role in the performance and to the amount of power assigned to each user sharing the same RB [8], such that users coupled with distinctive channel conditions where the user with a bad channel condition allocated with higher power level than the user with good channel condition. One of the main designing issues to consider is the resource allocation in NOMA that can be identified by user pairing and power allocation. User pairing helps to identify the perfect couple of users to share a single $\mathrm{RB}$ while power allocation divide the power among the users sharing this RB. The optimal approach reached through the brute force strategy where all the possible solutions of user pairs and power allocation coefficients searched. Though the optimal performance gained, it is highly complex not to mention the complexity of SIC performance and excessive signaling overhead [9]. Reducing SIC execution can be established through the user pairing scheme that widely studied in different researches over the past years. Another designing aspect of downlink NOMA is power allocation which in addition helps boosting the performance of the system due the power domain multiplexing strategy. In [10], a general overview of downlink NOMA and a comparative simulation of different user pairing and power allocation schemes maintained. Random user pairing and channel state sorting based user pairing evaluated with fixed power allocation (FPA), through the simulation channel state sorting based user pairing achieves higher system sum rate than random user pairing. On the other hand, Full Search Power Allocation (FSPA) proven to reach optimal performance of the system though it is highly complex. Therefore, it is strictly essential to understand the tradeoff between the system performance and 
the complexity of the system. Resource allocation received great attention from research society but optimal resource allocation still a very challenging task. In the downlink and uplink NOMA system, user clustering and power allocation were studied in [11]. Considering the channel gain difference a near-optimal user clustering algorithm proposed that aim to maximize the sum rate, for each cluster, an optimal power allocation strategy is given. The author in [12], propose iterative water-filling power allocation used with a greedy algorithm based user pairing to maximize users rate. Optimization of user pairing using matching theory proposed in [13], where a game between user and subchannel is estimated to match users into subchannels to maximize the sum rate of the system. Matching problem-based subchannel allocation and DC programming power allocation across subchannels and between users in each subchannel to boost energy efficiency studied in [14]. In [15], a bisection based iterative algorithm proposed for solving the non-convex problem yield from power allocation, the main objective of the proposed algorithm is to enhance the fairness of the system.

Genetic algorithm (GA) was introduced as a programming technique based on biological evolution [16]. It is characterized by overwhelming searching capability, which is usually utilized to find the optimal solution for complex problems. GA is employed for multiple domains of interest such as: data mining [17], fault diagnoses [18], cloud computing [19], Wireless Sensor Networks (WSN) [20], where cellular networks are no exception. In the LTE OFDMA system, GA utilized to learn antennas coverage pattern which leads to enhance the capacity of the system and decrease the network interference [21]. In the downlink NOMA system, a resource allocation algorithm using GA is proposed for pairing users that share the same frequency resource with an optimal power allocation strategy [22], results show that through the proposed algorithm a fast coverage to the target solution is achieved. On the other hand, GA utilized for power allocation in [23]. The proposed GA power allocation algorithm aims to maximize the achievable sum rate, results show that GA based NOMA overcome the performance of OMA. From the discussed works, reaching optimality in power allocation is still very challenging task especially with higher number of users sharing a single RB. Therefore, GA adopted for power allocation to maximize the system's sum rate considering power conditions and QoS of users which is assumed in this work as the minimum user's data rate.

The rest of the paper organized as follows: Section II discuss the mathematical model of the system. Power allocation problem formulated in Section III where the next Section IV presents the proposed power allocation algorithm. In Section $\mathrm{V}$, the performance scenarios and performance metrics are evaluated to simulate, analyze, and compare performance. Finally, Section VI represents the conclusion and future works.

\section{SYSTEM MODEL}

In a single cell, we study a downlink Multiuser NOMA with one Base Station (BS) and an arbitrary set of K users (k€

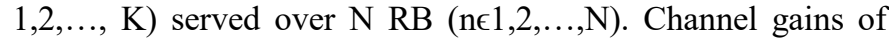
allocated users to nth RB ordered as $\left|h_{1, n}\right| \geqslant\left|h_{2, n}\right| \geqslant\left|h_{k, n}\right| \geqslant \ldots \geqslant$ $\left|h_{K, n}\right|$, such that channel gain utilized to define the transmission power for each user in the users set allocated to that RB. Nevertheless, power allocation, user pairing, and SIC decoding order depend on channel gain sequence. At receiver side, the scheduled strong user on the nth RB uses SIC to exclude interuser interference which is estimated through decoding other multiplexed signals of other users messages and subtracting these signals to be able to decode the signal of its own message. Weak users on other hand decode their own signals treating other signals as an interference, this process have negligible degradation on the performance due to power allocation policy followed in NOMA systems where weak users associated with high power levels. In downlink system, BS multiplex the messages of users sharing $n^{\text {th }}$ RB via superposition coding, thus superimposed signal is expressed as:

$x_{n}=\sum_{i=1}^{M} \sqrt{P_{i, n}} S_{i}$

Considering the $R B$ total power $\sum_{i=1}^{m} p_{i, n}=p_{s}$ where $p_{i, n}$ denote the power coefficient for $\mathrm{UE}_{\mathrm{i}}$ in $\mathrm{RB}_{\mathrm{n}}$. The system total power is $\sum_{j=1}^{\mathrm{N}} \mathrm{p}_{\mathrm{j}}=\mathrm{p}_{\mathrm{t}}$, where $\mathrm{n}$ and $\mathrm{m}(\mathrm{m} \epsilon 1,2, \ldots, M)$ represent the index of RB and the index of users multiplexed over a RB, respectively. Assuming perfect knowledge of the channel state information of all users and Additive White Gaussian Noise (AWGN) channel is considered, user's $\mathrm{k}$ received signal on $\mathrm{RB}_{\mathrm{n}}$ is calculated by:

$y_{k, n}=\sqrt{P_{k, n}}\left|h_{k, n}\right| s_{k}+\sum_{i=1, i \neq k}^{M} \sqrt{P_{i, n}}\left|h_{k, n}\right| s_{i}+V_{k, n}$

where the signals $s_{k}$ and $s_{i}$ is multiplexed over $\left|h_{k, n}\right|$ that represents the channel attenuation factor between user $\mathrm{k}$ and BS on $\mathrm{RB}_{\mathrm{n}}$. $\mathrm{V}_{\mathrm{k}, \mathrm{n}}$ is the power spectral density with AWGN $\mathrm{N}_{0}$ (W/Hz). Total bandwidth is divided equally among RB such that the bandwidth of a specific RB is defined as $B_{n}=\frac{B}{N}$. The total power on all RBs is assumed to be equivalent thus Signal Interference to Noise Ratio (SINR) for user $K$ in $R_{n}$ is represented as [24]:

$\operatorname{SINR}_{K, n}=\frac{p_{K, n}\left|h_{K, n}\right|}{N_{0} B+\sum_{i=1}^{K-1} p_{i, n}\left|h_{K, n}\right|}$

$\mathrm{UE}_{\mathrm{K}}$ as the weakest user allocated in $\mathrm{RB}_{\mathrm{n}}$ do not perform SIC where the signals of other users allocated in the same RB and the environmental noise treated as an equivalent noise. In contrast $\mathrm{UE}_{\mathrm{k}}$, based on NOMA concept, performs SIC which enable successful decoding and subtracting $\left(\mathrm{UE}_{\mathrm{k}+1}, \mathrm{UE}_{\mathrm{k}+2}, \ldots \ldots\right.$, $\left.\mathrm{UE}_{\mathrm{K}}\right)$ message signals on while treat other $\left(\mathrm{UE}_{1}, \ldots, \mathrm{UE}_{\mathrm{k}-2}\right.$, $\mathrm{UE}_{\mathrm{k}-1}$ ) message signals and the environmental noise as an equivalent noise. Thus SINR for user $\mathrm{k}$ in $\mathrm{RB}_{\mathrm{n}}$ is expressed as:

$\operatorname{SINR}_{k, n}=\frac{p_{k, n}\left|h_{k, n}\right|}{N_{0} B+\sum_{i=1}^{k-1} p_{i, n}\left|h_{k, n}\right|}$ 
Assuming $M=2$, two users can concurrently share a $R B_{n}$. $\mathrm{R}_{1, \mathrm{n}}$ and $\mathrm{R}_{2, \mathrm{n}}$ represent user1 rate and user2 rate in $\mathrm{RB}_{\mathrm{n}}$ and are calculated as follow:

$R_{1, n}=B_{n} \log _{2}\left(1+\frac{p_{1, n} h_{1, n}}{N_{0} B_{n}}\right)$

$R_{2, n}=B_{n} \log _{2}\left(1+\frac{p_{2, n} h_{2, n}}{p_{1, n} h_{2, n}+N_{0} B_{n}}\right)$

where $\left|\mathrm{h}_{1, \mathrm{n}}\right| \geqslant\left|\mathrm{h}_{2, \mathrm{n}}\right|, \mathrm{UE}_{2, \mathrm{n}}$ do not perform SIC. On the other hand, $\mathrm{UE}_{1, \mathrm{n}}$ needs to extract $\mathrm{UE}_{2, \mathrm{n}}$ signal then decode its own signal. Generally, under a successful decoding and no error propagation system with a randomized inter-cell interference that can be seen as white noise, where the power coefficient of $\mathrm{UE}_{2, \mathrm{n}}$ given higher ratio than $\mathrm{UE}_{1, \mathrm{n}}$ such as $\mathrm{p}_{1} \leqslant \mathrm{p}_{2}$. Therefore, the achievable throughput of user $\mathrm{k}$ on $\mathrm{RB}_{\mathrm{n}}$ is expressed as:

$R_{k, n}=B_{n} \log _{2}\left(1+\frac{p_{k, n}\left|h_{k, n}\right|}{N_{0} B_{n}+\sum_{j=1}^{m-1} p_{j, n}\left|h_{k, n}\right|}\right)$

Whereas, the total system sum rate equals to the summation of total sum rate calculated over the RBs, which is represented as:

$R=\sum_{n=1}^{N} R_{n}=\sum_{n=1}^{N} \sum_{k=1}^{K} R_{k, n}$

It is worthy to mention that not only exclusive sum rate optimization of the system is provided, a spectral efficiency and energy efficiency performance metrics is also simulated. For each RB in NOMA system given the total sum rate calculated at the $R_{n}$ as $R_{n}$ and the bandwidth of this $R_{n}$ is $B_{n}$, then the spectral efficiency of the regarded $R_{n}$ is expressed as:

$S E_{n}=\frac{R_{n}}{B_{n}}$

Thus, the total spectral efficiency or as regarded as the system's spectral efficiency is calculated by:

$S E=\sum_{n=1}^{N} S E_{n}$

Additionally, energy efficiency over $\mathrm{RB}_{\mathrm{n}}$ given that $\mathrm{p}_{\mathrm{s}}$ and $\mathrm{p}_{\mathrm{c}}$ as the total RB power and the additional circuit power consumption, respectively. Therefore, energy efficiency of the $\mathrm{RB}_{\mathrm{n}}$ is defined as:

$E E_{n}=\frac{R_{n}}{p_{s}+p_{c}}$

where the total energy efficiency or as known by system's energy efficiency is expressed as:

$E E=\sum_{n=1}^{N} E E_{n}$

Due to the effect of the factor of dividing the power to paired users, an optimization of power allocation is needed. Additionally, most of the works presented to optimize power allocation consider two user multiplexing strategy according to simplicity, rather multiple user multiplexing strategy need to be researched. Motivated by that, the main contribution of this work is to propose a power allocation algorithm that maximize system's sum rate, the problem related to the power allocation formulated in the next section followed by the solution approach.

\section{PROBLEM StATEMENT}

In this section, we formulate a maximization of system's sum rate power allocation as an optimization problem. In order to enhance system's sum rate, it is important for each user to reach or exceed a minimum rate. Therefore, power allocation optimization problem is formulated as:

$\max _{\mathrm{p}_{\mathrm{m}, \mathrm{n}}} \sum_{\mathrm{n}=1}^{\mathrm{N}} \mathrm{R}_{\mathrm{n}}$

Subject to:

C1: $\mathrm{R}_{\mathrm{k}, \mathrm{n}} \geq \mathrm{R}_{\min }$

C2: $\sum_{\mathrm{m}=1}^{\mathrm{M}} \mathrm{p}_{\mathrm{m}, \mathrm{n}}=\mathrm{p}_{\mathrm{s}}$

C3: $\mathrm{p}_{\mathrm{m}, \mathrm{n}}>0$

Where $R_{\text {min }}$ represents the minimum user rate requirement, constraint $\mathrm{C} 1$ applies that each user data rate have to reach or exceed a specific user rate. Power allocation constraints discussed in $\mathrm{C} 2$ and $\mathrm{C} 3$, where the available power of an individual $\mathrm{RB}$ divided among the users sharing it as expressed in $\mathrm{C} 2$ such that the summation of power coefficients equals to total power of the RB. C3 on other hand indicates that power allocation coefficient must be higher than zero.

\section{GENetic Algorithm BASEd POWER AllocAtion} (GAPA)

Genetic algorithm (GA) adopted as an intelligent search algorithm to find the optimal solution, is defined as an optimization method that explores huge search space based on a powerful meat-heuristic. To solve the power allocation problem formulated in the previous section, the Genetic Algorithm based Power Allocation (GAPA) processes are shown in Algorithm 1. The process of resource allocation performed iteratively beginning by pairing users to a specific RB then GAPA is utilized. Therefore, the processes of GAPA held where the number of genes in chromosomes is highly dependent on the multiplexing number such that for two users sharing the same RB (scenario1), the genes of chromosomes in the generations is equivalent to 2. Moreover, considering the case of three users multiplexing genes of chromosomes in every generation is equal to 3 also. Due to the nature of solutions, a string of real number representation was adopted such that the power level of an individual user presented in real numbers.

A random set of $\mathrm{L}$ chromosomes are generated, each representing a feasible solution. For some cases, the coverage of the population was maintained without a limitation in size. Rather in this work, we limit the number of chromosomes in a generation to $\mathrm{L}=100$ and the number of generations to be 50 . Chromosomes of each generation including the initial generation evaluated based on a predefined fitness function represent the optimization objective, by that means the fitness function in this paper is the sum rate formulated in (8). Then generations of chromosomes go through other GA operations of selection, crossover, and mutation to explore and invoke modified solutions. 


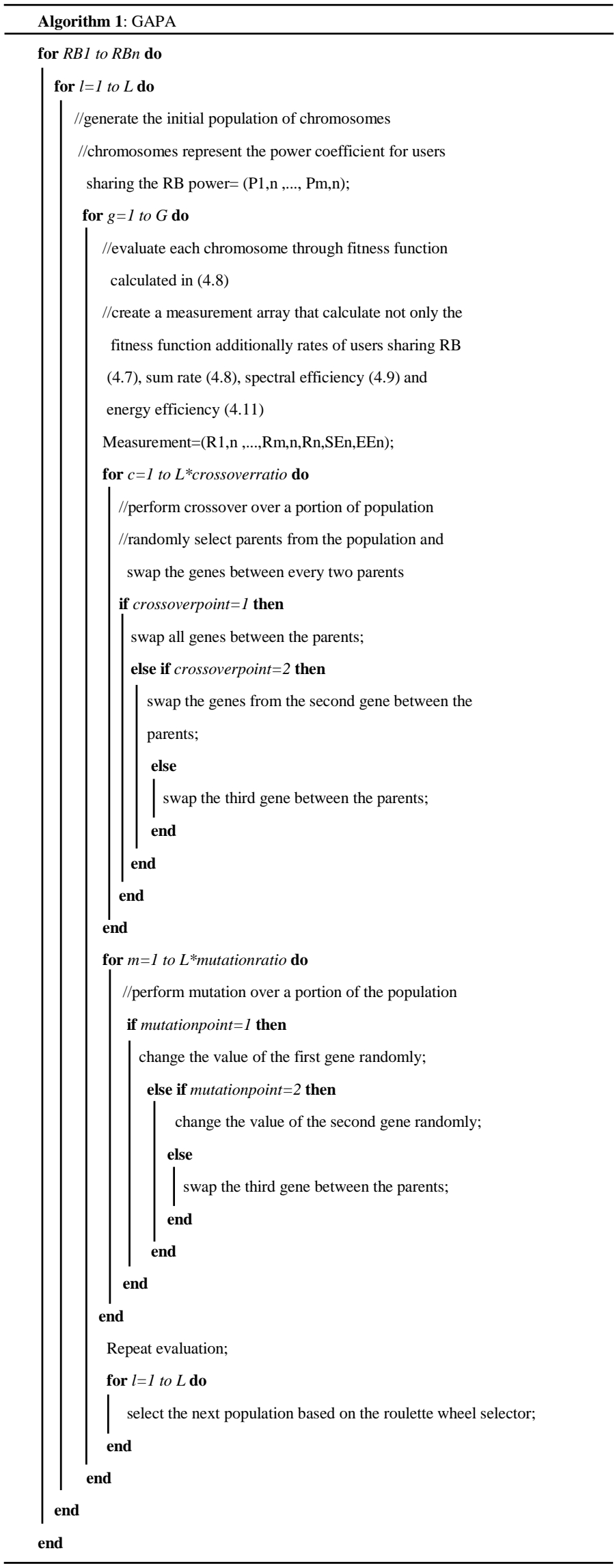

Through crossover and mutation, or as known by reproduction, a new offspring of chromosomes are generated where crossover ratio is assumed to be 0.5 and mutation ratio equals to 0.25 . A $50 \%$ of the current population perform crossover where two arbitrary selected chromosomes referred to as parents swap their genes such that in GAPA, crossover have to consider the main requirements such that after swapping the genes among two chromosomes power levels of users with higher channel gain should not be higher than the power level of users with lower channel gain. Another point to be taken into consideration that the summation of power levels after exchanging the genes should be equal to the total RB power based on the second condition of the formulated problem, where a one point crossover is applied. The process of crossover is based on the number of genes summarized in Table I.

TABLE I. CROSSOVER OPERATION IN GAPA

\begin{tabular}{|c|c|c|c|c|c|c|c|}
\hline $\begin{array}{l}\text { Crossover } \\
\text { cases }\end{array}$ & $\begin{array}{l}\text { Two } \\
\text { (scena } \\
\text { M=2 }\end{array}$ & $\begin{array}{l}\mathrm{r} \mathbf{~ m t} \\
\text { 1) }\end{array}$ & & $\begin{array}{l}\text { Three } \\
\text { (scena } \\
\text { M=3 }\end{array}$ & $\begin{array}{l}\text { Iser } \\
\text { io } 2\end{array}$ & Itip & \\
\hline \multirow{5}{*}{ Case 1} & \multicolumn{3}{|c|}{ cuturer } & \multicolumn{4}{|c|}{ ontritest } \\
\hline & \multirow{2}{*}{$\begin{array}{l}\text { nuel } \\
\text { nuse: }\end{array}$} & pi & F. & \multirow{2}{*}{$\begin{array}{l}\text { ntext1 } \\
\text { nust }\end{array}$} & A & 12 & Ft \\
\hline & & $\beta$ & $F_{2}$ & & ${ }^{\prime}$ & 12 & $\beta$ \\
\hline & \multirow{2}{*}{$\begin{array}{l}\text { atsent } \\
\text { athert? }\end{array}$} & $\beta_{1}$ & $F_{7}$ & \multirow{2}{*}{$\begin{array}{l}\text { shesi } \\
\text { stonti }]\end{array}$} & A & $\mathrm{n}$ & $F$ \\
\hline & & $\vec{p}$ & F: & & $n$ & P: & b. \\
\hline \multirow{5}{*}{ Case 2} & \multicolumn{3}{|c|}{$\frac{1}{1}$} & \multicolumn{4}{|c|}{ Ganx: } \\
\hline & \multirow[b]{2}{*}{ men: } & 3 & $b_{i}$ & \multirow{2}{*}{$\begin{array}{l}\text { heit [ } \\
\text { metz [ }\end{array}$} & $s_{1}$ & $\hbar$ & 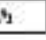 \\
\hline & & 3 & Fi & & 3 & it & s \\
\hline & \multirow{2}{*}{ 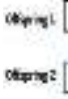 } & 3 & r. & \multirow{2}{*}{ 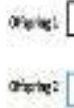 } & in & 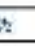 & $s$ \\
\hline & & $B_{1}$ & fi & & B & z & 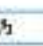 \\
\hline \multirow{5}{*}{ Case 3} & & & & \multicolumn{4}{|c|}{ Geoser: } \\
\hline & & & & Avert & $f$ & $i$ & in \\
\hline & & & & tron:2 & $\mathrm{St}_{\mathrm{H}}$ & $i$ & $\hat{\mathrm{h}}$ \\
\hline & & & & $a \times \times 81$ & $f_{1}$ & in & in \\
\hline & & & & aher:?2 & $H_{1}$ & is & in \\
\hline
\end{tabular}

On the other hand, $25 \%$ of chromosomes in a population chosen for mutation where random chromosomes are selected such that for the randomly chosen gene the value will be altered. Based on the dependability of the solved problem to real numbers a uniform mutation is utilized. Therefore, the altered value on a specific gene is randomly generated such that it should not exceed an upper bound or not be less than a lower bound which in our case determined by the total 
transmission power on a RB such that by adding the power ratios assigned to each user associated in this $R B$ the value should be equal to the total RB power. Selection then held which plays a huge role in forming the new generations, from different approaches for selection a roulette wheel selection is employed which is based on a probability distribution that gives the probability of selection to each chromosome. Thus, the probability of selection for chromosome $\mathrm{j}$ is defined as:

$B_{j}=\frac{R_{j}}{\sum_{i=1}^{L} R_{i}}$

These processes go in a cycle until the stopping criteria is reached which is assumed to be the number of generations that assumed earlier to be 50. Therefore, user pairing and GAPA performed iteratively for each RB available in the system.

\section{RESULTS AND DISCUSSION}

For the simulation results, the performance of our proposed power allocation algorithm is compared with FSPA that present an optimal solution. The simulation runs into a NOMA system with one BS. A single transmitting antenna and multiple users, each is occupied with one receiver antenna in a single cell. System's sum rate, spectral efficiency, and energy efficiency are evaluated for GAPA with exhaustive search user pairing in downlink NOMA system. Through this simulation, we assume the channel to be the product of free-space path loss and Gaussian white noise. Table II presents the most commonly used simulation parameters that is used commonly in similar researches. RBs are characterized with equivalent amount of bandwidth and downlink transmission power. For this scenario, the number of users in the system is considered to be 6 and 12 due to the high complexity produced by the large number of users in the system. We set the minimum rate that can be achieved by each user $R_{\min }$ to $100 \mathrm{kbps}$. In addition the number of GA individuals, crossover ratio, mutation ratio, and the number of generations as a stop criteria are set to $100,0.50$, $0.25,50$, respectively.

In the beginning, the performance of the system with different number of users served among the cell is simulated. Therefore, the number of users are assumed to be either $\mathrm{K}=6$ or $\mathrm{K}=12$. Both cases are evaluated with GAPA and FSPA for power allocation and exhaustive search user pairing, with three users multiplexed per $\mathrm{RB}(\mathrm{M}=3)$. That implies with $\mathrm{K}=6$, the number of RBs needed for users to be served among equals $\mathrm{N}=2$. On other hand, with $\mathrm{K}=12$ the number of $\mathrm{RBs}$ for users to be paired on as a triple is $\mathrm{N}=4$.

A comparison between FSPA and GAPA as a function of the system's sum rate is illustrated in Fig. 1. The figure shows that with the increase in the transmitted power, the gap between FSPA and GAPA increase for both downlink NOMA systems. Considering the system with six users $(K=6)$, both power allocation algorithms reaching the same performance at lower transmitted power while GAPA outperforms FSPA for higher transmitted power due to the heuristic searching method. On the other hand, in downlink NOMA with twelve users $(K=12)$ GAPA achieves superior system's sum rate than FSPA for higher transmitted power where on lower transmitted power FSPA is better though GAPA is low complex.
TABLE II. SIMULATION PARAMETERS

\begin{tabular}{|c|c|}
\hline Parameter & Value \\
\hline Transmitted Power $\left(\mathrm{p}_{\mathrm{t}}\right)$ & $316 \mathrm{~mW}(25 \mathrm{dBm})$ \\
\hline Total Bandwidth (B) & $5 \mathrm{MHz}$ \\
\hline Number of resource blocks RPs (N) & 24 \\
\hline Number of subcarrier & 12 per $\mathrm{RP}$ \\
\hline Noise Spectral Density $\left(\mathrm{N}_{0}\right)$ & $-150 \mathrm{dBw} / \mathrm{Hz}$ \\
\hline Channel estimation & Ideal \\
\hline Channel & AWGN \\
\hline Traffic Model & Full Buffer \\
\hline Circuit power $\left(\mathrm{p}_{\mathrm{c}}\right)$ & $1 \mathrm{w}$ \\
\hline Multiplexed users over single RB (M) & 2,3 \\
\hline Number of users (K) & 6,12 \\
\hline Number of transmit antenna at BS & 1 \\
\hline Number of receiver antenna at UE & 1 \\
\hline Minimum user's data rate $\left(\mathrm{R}_{\min }\right)$ & $100 \mathrm{kbps}$ \\
\hline Number of chromosomes per generation (L) & 100 \\
\hline Number of generations (G) & 50 \\
\hline Crossover ratio & 0.50 \\
\hline Mutation ratio & 0.25 \\
\hline
\end{tabular}

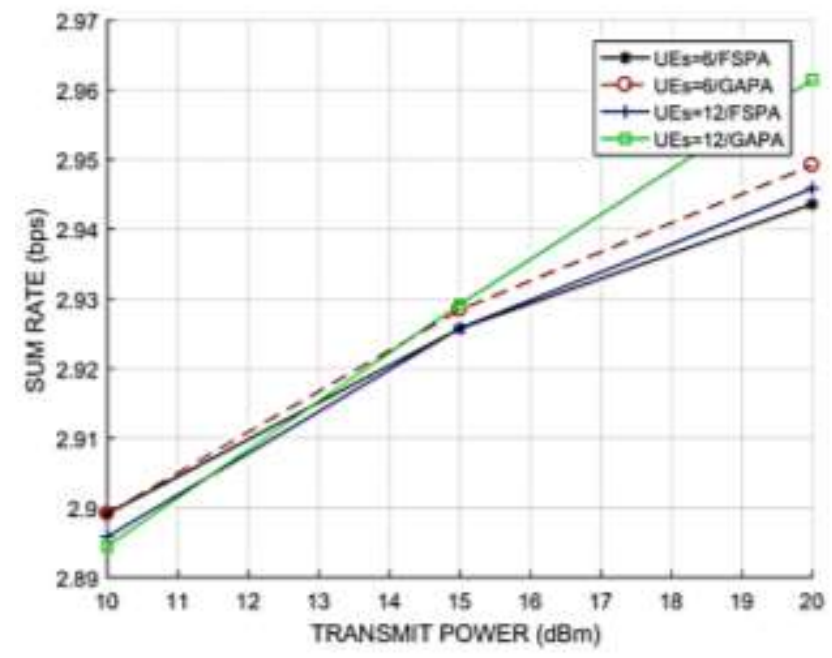

Fig. 1. Sum Rate Performance of 3-users Multiplexing Downlink NOMA $(\mathrm{M}=3)$ with FSPA and GAPA.

Fig. 2 and Fig. 3 illustrate the performance of the system based on spectral efficiency that is expressed as the ratio of sum rate and bandwidth. In downlink NOMA with $\mathrm{K}=6$, GAPA performs better than FSPA especially with higher transmitted power as shown in Fig. 2. Additionally, Fig. 3 illustrates the performance of spectral efficiency in downlink NOMA system with $\mathrm{K}=12$. Searching based on heuristics benefit with the system's performance such that GAPA is more spectrally efficient than FSPA. Number of users served in the system effect the performance where with the growth in the number of users the spectral efficiency increase. 


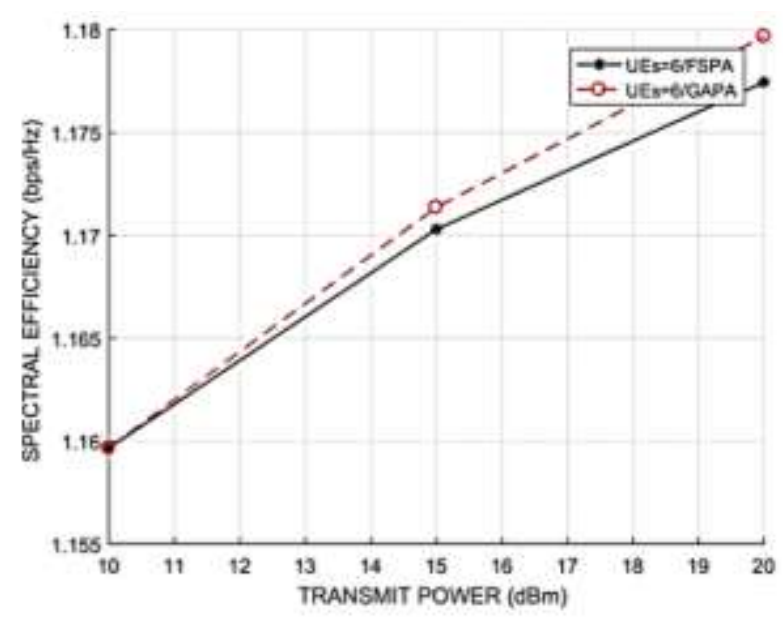

Fig. 2. Spectral Effeciency Performance of 6-users (K=6) Downlink NOMA with FSPA and GAPA.

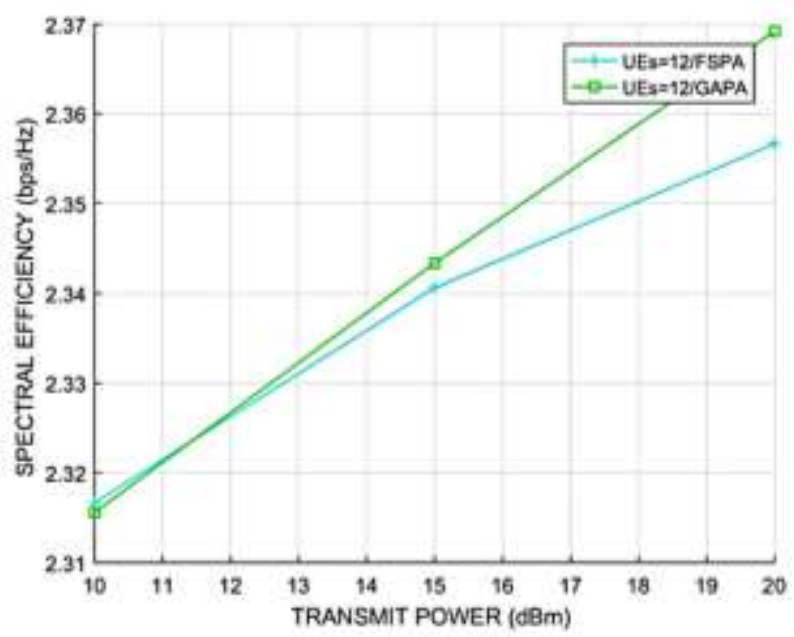

Fig. 3. Spectral Effeciency Performance of 12-users $(K=12)$ Downlink NOMA with FSPA and GAPA.

Energy efficiency (EE) of the system is presented in Fig. 4. The value of $P_{c}$ is assumed to be constant and equal to $1 \mathrm{~W}$. Results shows that both FSPA and GAPA have reached an equivalent performance based on overall system energy efficiency for 6 users case. In contrast, downlink NOMA with $\mathrm{K}=12$ GAPA has consumed less energy than FSPA due to the utilized searching technique. It is also found that with the increase in transmission power system, the energy efficiency decreased due to transmission with large power to maximize overall sum rate. Referring to Fig. 1, GAPA with 12 users gain has the highest system sum rate which lead to deceased system EE.

Time complexity of simulated power allocation algorithms in both systems is evaluated. Fig. 5 shows that GAPA in both system decreases the complexity of the system with higher performance gain than FSPA that represent the best optimum performance. FSPA used in downlink NOMA with $\mathrm{K}=12$ takes the longest time where with GAPA the time complexity decreased significantly. Additionally, in downlink NOMA with $\mathrm{K}=6$ FSAP reach higher complexity in GAPA.

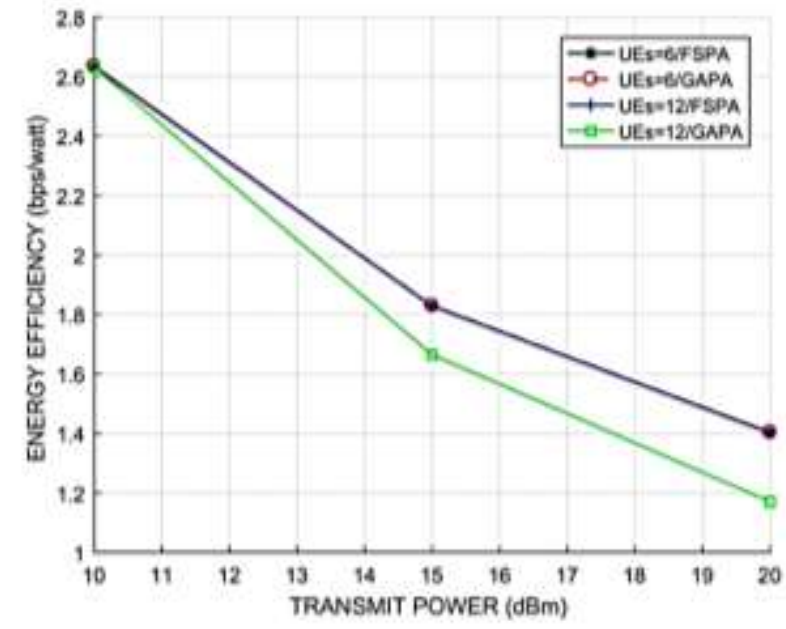

Fig. 4. Energy Effeciency Performance of Downlink NOMA with FSPA and GAPA.

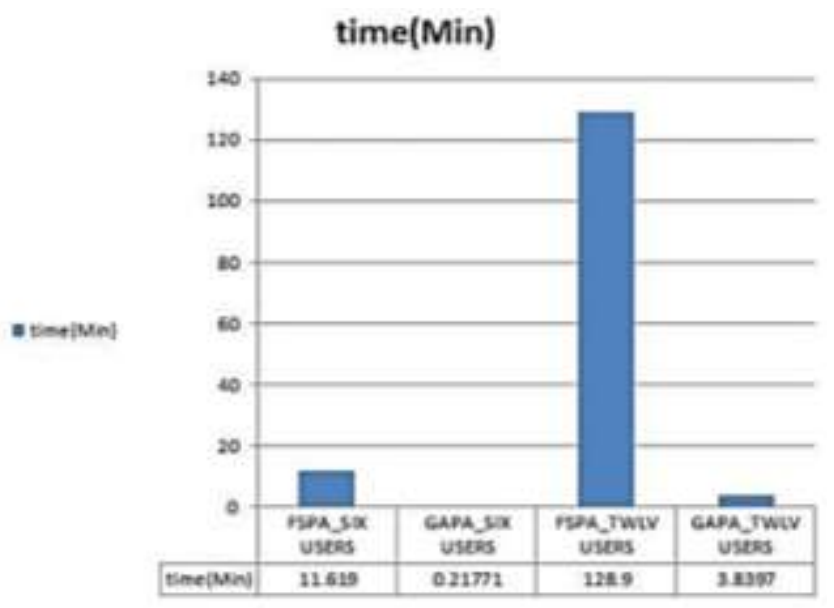

Fig. 5. Time Complexity of Power Allocation Algorithm in Downlink NOMA Systems.

A second scenario for simulation is done through a simulation of different resource allocation schemes, where we assume that these schemes differentiated by the user pairing algorithms. Three user pairing schemes simulated with GAPA. A random user pairing that works by pairing arbitrary users into a cluster to be allocated into an individual RB, a channel state based sorting user pairing that is based on dividing the users based on their channel conditions into different NOMA clusters each must contain a strong and weak users. In addition, exhaustive search based user pairing is considered. All algorithms invoked with GAPA.

As illustrated in Fig. 6 to Fig. 8, channel state information based sorting user pairing gain the best performance among other algorithms in term of system sum rate, spectral efficiency and energy efficiency. Such that with the increase in transmit power the performance gain of the three user pairing algorithms increase as well. System's sum rate and overall spectral efficiency performance of exhaustive approach are slightly higher than random user pairing, where in term of energy efficiency the performance of these two user pairing algorithms are equivalent. 


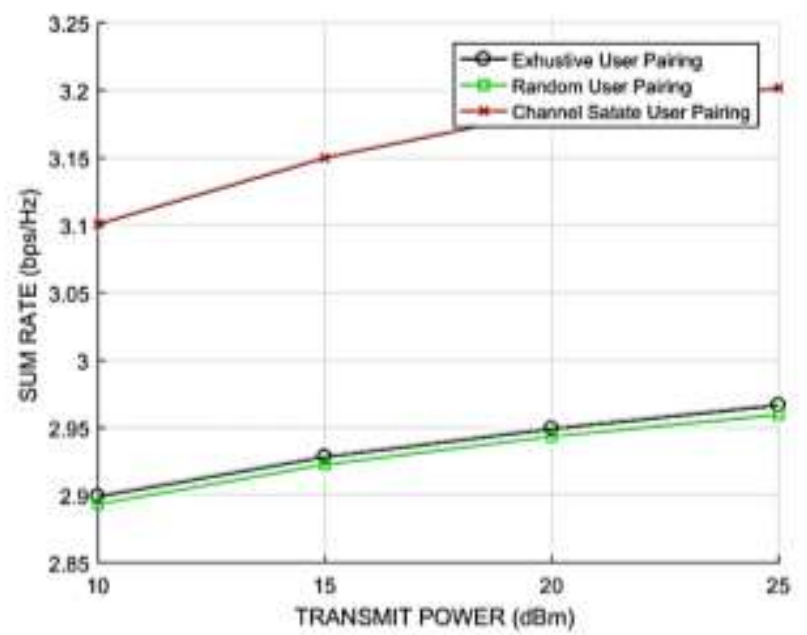

Fig. 6. Sum Rate Performance of 3-users Multiplexing Downlink NOMA $(\mathrm{M}=3)$ with Different User Pairing Algorithms.

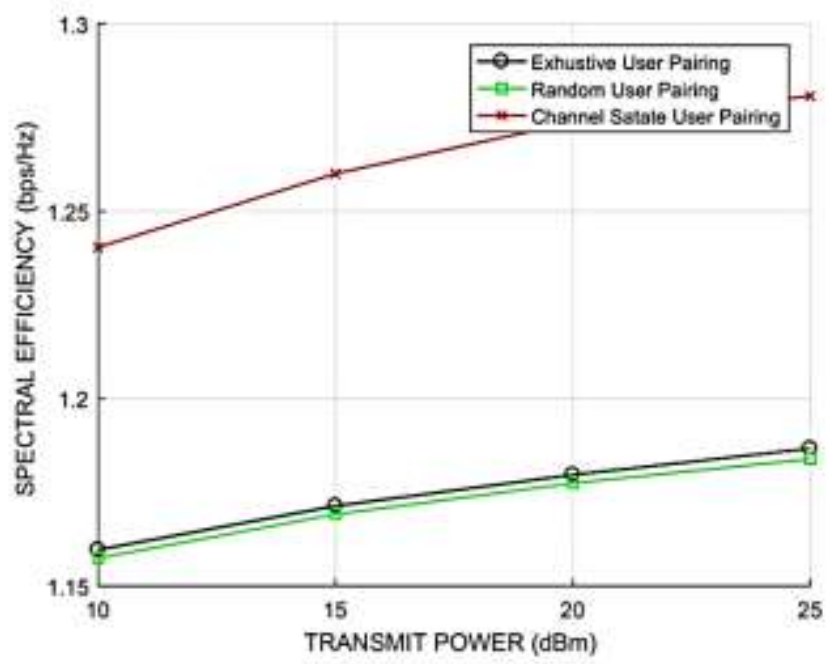

Fig. 7. Spectral Efficiency Performance of 3-users Multiplexing Downlink NOMA (M=3) with Different User Pairing Algorithms.

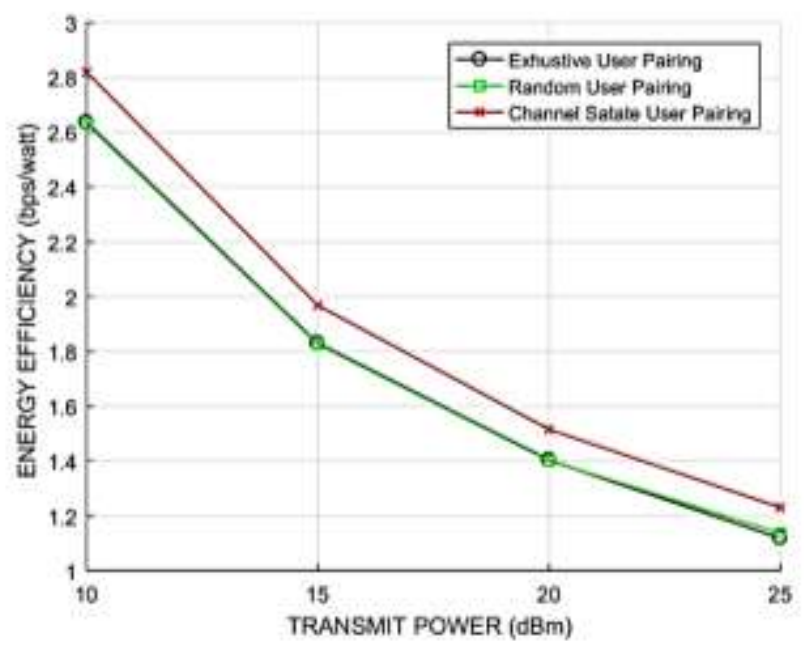

Fig. 8. Energy Efficiency Performance of 3-users Multiplexing Downlink NOMA (M=3) with Different User Pairing Algorithms.
Finally, in downlink NOMA with $\mathrm{K}=12$ the effect of the number of users multiplexed over the same radio resource is investigated. For evaluation, two multiplexing scheme $M=2$ and three multiplexing scheme $\mathrm{M}=3$ for downlink NOMA system is simulated. Therefore, the number of RB needed for tow multiplexing scheme $\mathrm{M}=2$ given as $\mathrm{N}=6$. Where on the case with three user multiplexing scheme $\mathrm{M}=3$, four $\mathrm{RBs} \mathrm{N}=4$ needed. The result in Fig. 9 shows that the sum rate of the system with three users multiplexing system $\mathrm{M}=3$ overcome system's sum rate with two user multiplexing scheme $M=2$. Therefore, increasing the number of users sharing the same resource helps to increase system's sum rate performance. In addition, utilization of GAPA with both multiplexing schemes scenarios increase the performance of the system especially with higher transmitted power levels.

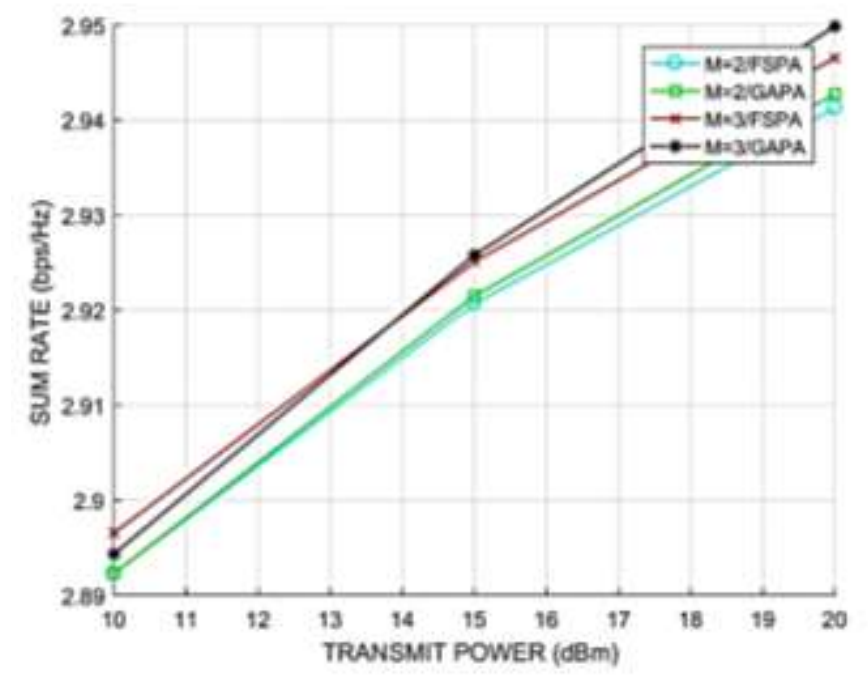

Fig. 9. Sum Rate Performance of 2-users and 3-users Multiplexing Downlink NOMA ( $M=2$ and $M=3)$.

\section{CONCLUSION}

Next generation cellular networks needs a new and highly effective technologies to be adopted, one of the promising recommended technology for radio accessing technique is NOMA. The basic concept of NOMA is to utilize power as its new diminution. In a downlink NOMA system, power allocation optimization problem is formulated where total subchannel power and minimum user's data rate are taken in consideration. Genetic algorithm power allocation (GAPA) is proposed to solve the problem, which can not only achieve high performance gain but can decrease the complexity. GAPA is utilized with an exhaustive user pairing scheme and is evaluated through comparing the performance with FSPA. Results show that the proposed algorithm reach a good performance in addition the complexity is decreased. GAPA outperform FSPA with large number of users and high transmission power. Moreover, exhaustive, random, and channel state based sorting user pairing algorithms were invoked with GAPA. Through these experiments, channel state based user pairing overcome the other two user pairing algorithms based on system's sum rate, spectral efficiency, and energy efficiency. Finally, the impact of multiplexed users in a single subchannel was studied, where results revealed that with 
higher number of multiplexed users, the system's sum rate increased. For future investigations, enabling the proposed algorithm with a downlink system with imperfect channel state information and further enhancement of the spectral efficiency by adding MIMO technology needed to be studied.

\section{REFERENCES}

[1] Kalhoro, S., Umrani, F. A., Khanzada, M. A., \& Rahoo, L. A. (2019). Matched Filter Based Spectrum Sensing Technique for 4G Cellular Network. Mehran University Research Journal of Engineering and Technology, 38, 373-978. https://doi.org/https://doi.org/10.22581/muet1982.1904.10.

[2] Vamvakas, P., Tsiropoulou, E. E., \& Papavassiliou, S. (2019). Dynamic Spectrum Management in 5G Wireless Networks: A Real-Life Modeling Approach. In IEEE INFOCOM 2019 - IEEE Conference on Computer Communications. Paris, $\quad$ France,: https://doi.org/10.1109/INFOCOM.2019.8737443.

[3] Vaezi, M., \& Poor, H. V. (2019). NOMA: An Information-Theoretic Perspective. In Multiple Access Techniques for 5G Wireless Networks and Beyond (pp. 167-193). Springer, Cham. https://doi.org/https://doi.org/10.1007/978-3-319-92090-0_5.

[4] Saito, Y., Kishiyama, Y., Benjebbour, A., Nakamura, T., Li, A., \& Higuchi, K. (2013). Non-orthogonal multiple access (NOMA) for cellular future radio access. In 2013 IEEE 77th Vehicular Technology Conference (VTC Spring) (pp. 1-5). Dresden. https://doi.org/10.1109/VTCSpring.2013.6692652.

[5] Agyapong, P. K., Iwamura, M., Staehle, D., Kiess, W., \& Benjebbour, A. (2014). Design considerations for a 5G network architecture. IEEE Communications Magazine, 52(11), 65-75. https://doi.org/10.1109/MCOM.2014.6957145.

[6] Liu, Y., Qin, Z., Elkashlan, M., Ding, Z., Nallanathan, A., \& Hanzo, L. (2017). Non-orthogonal multiple access for 5G and beyond. Proceedings of the IEEE, 105(12), 2347-2381. https://doi.org/10.1109/JPROC.2017.2768666.

[7] Choi, J. (2018). Throughput analysis for multiuser diversity of two users with SIC in NOMA systems. In 2018 International Conference on Signals and Systems (ICSigSys) (pp. 120-124). Bali. https://doi.org/10.1109/ICSIGSYS.2018.8372649.

[8] Ding, Z., Peng, M., \& Poor, H. V. (2015). Cooperative Non-Orthogonal Multiple Access in 5G Systems. IEEE Communications Letters, 19(8), 1462-1465. https://doi.org/10.1109/LCOMM.2015.2441064.

[9] Benjebbour, A., Saito, Y., Kishiyama, Y., Li, A., Harada, A., \& Nakamura, T. (2013). Concept and practical considerations of nonorthogonal multiple access (NOMA) for future radio access. In 2013 International Symposium on Intelligent Signal Processing and Communication Systems (pp. 770-774). Naha. https://doi.org/10.1109/ISPACS.2013.6704653.

[10] Alghasmari, W. F., \& Nassef, L. (2020). Power Allocation Evaluation for Downlink Non-Orthogonal Multiple Access (NOMA). International Journal of Advanced Computer Science and Applications(IJACSA), 11(4). https://doi.org/10.14569/IJACSA.2020.0110417.

[11] Ali, M. S., Tabassum, H., \& Hossain, E. (2016). Dynamic User Clustering and Power Allocation for Uplink and Downlink NonOrthogonal Multiple Access (NOMA) Systems. IEEE Access, 4, 63256343. https://doi.org/10.1109/ACCESS.2016.2604821.
[12] Cai, W., Chen, C., Bai, L., Bai, Y., \& Choi, J. (2017). Subcarrier and power allocation scheme for downlink OFDM-NOMA systems. IET Signal Processing, 11(1). https://doi.org/10.1049/iet-spr.2016.0188.

[13] Di, B., Bayat, S., Song, L., \& Li, Y. (2015). Radio Resource Allocation for Downlink Non-Orthogonal Multiple Access (NOMA) Networks Using Matching Theory. In 2015 IEEE Global Communications Conference (GLOBECOM). San Diego, CA, USA: IEEE. https://doi.org/10.1109/GLOCOM.2015.7417643.

[14] Fang, F., Zhang, H., Cheng, J., \& Leung, V. C. M. (2016). EnergyEfficient Resource Allocation for Downlink Non-Orthogonal Multiple Access Network. IEEE Transactions on Communications, 64(9), 37223732. https://doi.org/10.1109/TCOMM.2016.2594759.

[15] Timotheou, S., \& Krikidis, I. (2015). Fairness for Non-Orthogonal Multiple Access in 5G Systems. IEEE Signal Processing Letters, 22(10), 1647-1651. https://doi.org/10.1109/LSP.2015.2417119.

[16] Kramer, O. (2017). Genetic Algorithm Essentials. Springer, Cham. https://doi.org/https://doi.org/10.1007/978-3-319-52156-5.

[17] Ting, C.-K., Wang, T.-C., Liaw, R.-T., \& Hong, T.-P. (2017). Genetic algorithm with a structure-based representation for genetic-fuzzy data mining. Soft Computing, 21, 2871-2882. https://doi.org/https://doi.org/10.1007/s00500-016-2266-z.

[18] Zhu, X., Xiong, J., \& Liang, Q. (2018). Fault Diagnosis of Rotation Machinery Based on Support Vector Machine Optimized by Quantum Genetic Algorithm. IEEE Access, 6, 33583-33588. https://doi.org/10.1109/ACCESS.2018.2789933.

[19] Mahmood, A., Khan, S. A., \& 3, R. A. B. (2017). Hard Real-Time Task Scheduling in Cloud Computing Using an Adaptive Genetic Algorithm. $\begin{array}{lll}\text { Computers 2018, 6(2), } & \end{array}$ https://doi.org/https://doi.org/10.3390/computers6020015.

[20] Jha, S. K., \& Eyong, E. M. (2018). An energy optimization in wireless sensor networks by using genetic algorithm. Telecommunication Systems, 67, 113-121. https://doi.org/https://doi.org/10.1007/s11235017-0324-1.

[21] Yang, X., Wang, Y., Zhang, D., \& Cuthbert, L. (2010). Resource Allocation in LTE OFDMA Systems Using Genetic Algorithm and Semi-Smart Antennas. In 2010 IEEE Wireless Communication and Networking Conference. Sydney,Australia: IEEE. https://doi.org/10.1109/WCNC.2010.5506423.

[22] Gemici, Ö. F., Kara, F., Hokelek, I., Kurt, G. K., \& Çırpan, H. A. (2017). Resource allocation for NOMA downlink systems: Genetic algorithm approach. In 2017 40th International Conference on Telecommunications and Signal Processing (TSP). Barcelona, Spain: IEEE.

[23] Ma, X., Wu, J., Zhang, Z., Zhang, Z., Wang, X., Chai, X., ... Dai, X. (2017). Power Allocation for Downlink of Non-orthogonal Multiple Access System via Genetic Algorithm. In 5GWN 2017 (pp. 459-470). Springer, Cham. https://doi.org/https://doi.org/10.1007/978-3-31972823-0_43.

[24] Hanif, M. F., Ding, Z., Ratnarajah, T., \& Karagiannidis, G. K. (2016). A Minorization-Maximization Method for Optimizing Sum Rate in the Downlink of Non-Orthogonal Multiple Access Systems. IEEE Transactions on Signal Processing, 64(1), 76-88. https://doi.org/10.1109/TSP.2015.2480042. 